\title{
Rosetta Asteroid Candidates
}

M.Antonietta Barucci

LESIA, Observatory of Paris

Marcello Fulchignoni

LESIA, Observatory of Paris

Mirel Birlan

LESIA, Observatory of Paris

Pierre Vernazza

LESIA, Observatory of Paris

Elisabetta Dotto

INAF, Osservatorio Astronomico di Roma

Alain Doressoundiram

LESIA, Observatory of Paris

\begin{abstract}
The new scenario of the Rosetta mission to comet $67 / \mathrm{P}$ ChuryumovGerasimenko (launch on February 2004), includes as baseline the fly-by of one or two asteroids. Several asteroids are now possible fly-by candidates (single or double) within the available resources. Other candidates whose fly-bys require a larger $\Delta \mathrm{v}$ can be also considered if the Rosetta interplanetary orbit insertion will cost less $\Delta \mathrm{v}$.

All the up to date available information on the possible targets are discussed in this report.
\end{abstract}

The new baseline of the Rosetta mission includes the fly-by of at least an asteroid (2009-2010) which will precede a long orbital rendez-vous with the $67 / \mathrm{P}$ Churyumov-Gerasimenko comet nucleus (2014). The selection of the asteroid target(s) depends on the $\Delta v$ available after the Rosetta probe interplanetary orbit insertion maneuver. A few $\mathrm{m} / \mathrm{sec} \Delta v$ are available for the asteroid science in the pre-launch resource budget; but there is the possibility to allocate to the asteroid some of the remnant $\Delta v$, now reserved as contingency for the insertion maneuver, as soon as the Rosetta probe will be on its way toward the comet. The idea is to wait the results (in terms of $\Delta v$ expenses) of the insertion maneuver and to consider the new available budget to perform the asteroid fly-by(s). For this reason it is necessary to be ready shortly after the beginning of the Rosetta interplanetary journey: an international observational campaign is started with 
the aim to increase at the maximum level the characterization of the possible asteroid targets of the mission.

This paper present the situation concerning the Rosetta asteroid targets choice as it is at the date of the IAU General Assembly in Sidney (July 2003). Ten main belt asteroids have been found allowing the Rosetta probe to have a single or even few double fly-bys whitin a $\Delta v$ range $10-150 \mathrm{~m} / \mathrm{s}$ (see table).

21 Lutetia is the largest object between these possible targets. On the basis of its visible spectra and IRAS albedo (Tedesco, 1992), it was classified as M type, but new observations in near-infrared and polarimetric data (Birlan et al. 2003) suggest that it is rather a C-type object similar to carbonaceous chondrite.

No data on 2181 Fogelin are available in literature. Considering its absolute magnitude the diameter can be estimated in the range $12-22 \mathrm{~km}$. We observed it with the IRTF/NASA on July 2003 and its near-infrared spectrum suggest a S type object (Birlan et al. 2003).

$\begin{array}{lccccc}\text { Asteroid } & \begin{array}{c}\text { Diam. } \\ (\mathrm{km})\end{array} & \begin{array}{c}\text { IRAS } \\ \text { albedo }\end{array} & \begin{array}{c}\text { Extra } \Delta \mathrm{v} \\ (\mathrm{km})\end{array} & \begin{array}{c}\text { Fly-by date } \\ \text { mm.dd.yy }\end{array} & \begin{array}{c}\text { Rel. Vel. } \\ (\mathrm{km} / \mathrm{s})\end{array} \\ \text { 21 Lutetia } & 96 & 0.22 \pm 0.02 & 131 & 07.10 .10 & 14.9 \\ \text { 437 Rhodia } & 13 & 0.70 \pm 0.08 & 87 & 09.17 .08 & 11.2 \\ \text { 1393 Sofala } & - & - & 113 & 09.11 .08 & 6.9 \\ \text { 1714 Sy } & - & 0.11 \pm 0.03 & 11 & 03.06 .08 & 8.2 \\ \text { 2181 Fogelin } & - & - & 19 & 05.25 .10 & 13.6 \\ \text { 2513 Baethsle } & 17 & 0.03 \pm 0.01 & 16 & 10.05 .08 & 8.6 \\ \text { 2867 Steins } & - & - & 61 & 09.06 .08 & 8.6 \\ \text { 3050 Carrera } & - & - & 74 & 07.31 .08 & 11.2 \\ \text { 3418 Izvekov } & 27 & 0.07 \pm 0.01 & 14 & 12.04 .10 & 11.3 \\ \text { 5538 Luichewoo } & - & - & 35 & 04.08 .09 & 5.6\end{array}$

The asteroid 437 Rhodia is an intriguing object due to its very high IRAS albedo. The synodical period of $\geq 56$ hours (Binzel, 1987) allow us to consider it within the slow rotator asteroid group.

If we consider the family members having spectra quite homogeneous (Florczak et al, 1998), we can assume that the asteroids 2513 Baethsle and 5538 Luichewoo belong to the $\mathrm{S}$ type which is characteristic of the members of the Flora family. IRAS albedo of 2513 Baethsle, very far from the typical S-type, may indicate its "interloper" character and its appurtenance to a more primitive (C or D) asteroid class. The asteroid 3418 Izvekov belongs to the C-type Themis family, this is in agreement with its IRAS albedo value. The visible spectrum of the asteroid 1393 Sofala (Xu et al, 1995) indicates a S-type composition.

The final decision on the asteroid candidates will be taken after the launch. Information on all the possible target asteroids is important to be able to contribute to the best choice of the targets and to optimise the mission science return.

21 Lutetia represents one of the most interesting candidate, in fact it's the only one which will allow us to obtain mass determination by radio science experiments, and consequently it will be possible to determine its density. Moreover, 
if the chondritic character of this object will be confirmed, it will cope with the scientific objectives of the mission: the exploration of the primitive bodies of the planetary system.

If Lutetia cannot be selected due to the lack of available $\Delta v$, the asteroid candidate choice has to be done favouring the objects characterized by: 1) the more primitive compositional types (C, P, D); 2 ) the slower fly-by relative velocity; and 3) the larger diameter.

To obtain all these information we urge the observer community to participate to the observational campaign of these objects during their 2004 opposition.

\section{References}

Binzel, R. P. 1987, Icarus, 72, 135.

Birlan, M., Barucci, M.A., Vernazza, V., Fulchignoni, M.,Binzel, R.P., Bus, S.J., Belskaia, I. and Fonasier, S. 2003, New Astronomy, 9, 343.

Florczak, M., Barucci, M.A., Doressoundiram, A., Lazzaro, D., Angeli, C. and Dotto, E. 1998, Icarus, 133, 233.

Xu, S., Binzel, R.P., Burbine, T.H. and Bus, S.J. 1995, Icarus, 115, 1.

Tedesco, E.F. 1992, PL-TR-92-2049. 